\title{
Darles rienda suelta a los instintos: la sexualidad para adolescentes hombres con experiencia de vida en calle, Medellín*
}

\author{
Giving instincts free rein: sexuality for male adolescents with experience of life on \\ the street, Medellin
}
"Dar rédea larga" aos instintos: A sexualidade para os adolescentes homens moradores de rua, Medellin

Álvaro Giraldo Pineda1'; Constanza Forero Pulido²; Anghie Phamela López Mejiäa; Luisa Fernanda Posada Romero4.

1 msc en Salud Pública, docente Facultad Nacional de Salud Pública Universidad de Antioquia. Colombia. alvaro.giraldo@udea.edu.co. Código ORCID: https://orcid.org/0000-0001-8942-6750.

2 msc en Salud Pública, Docente Facultad de Enfermería Universidad de Antioquia. Colombia. constanza.forero@udea.edu.co. ORCID: https://orcid.org/0000-0001-7494-6825.

3 Pregrado en Administración en Salud, Facultad Nacional de Salud Pública Universidad de Antioquia. Colombia. phamela.lopez2018@ gmail.com. ORCID: https://orcid. org/0000-0003-4385-6290.

$4 \quad$ Pregrado en Trabajo social Universidad de Antioquia. Colombia. luisaposada1474@gmail.com. oRcID: https://orcid.org/0000-00030119-2940.

Recibido: 19/02/2019. Aprobado: 17/10/2019. Publicado: 20/03/2020

Giraldo-Pineda Á, Forero-Pulido C; López-Mejía AP; Posada-Romero LF. Darles rienda suelta a los instintos: la sexualidad para adolescentes hombres con experiencia de vida en calle, Medellín. Rev. Fac. Nal. Salud Pública. 2020; 38 (2):e337097. Dor: https://doi.org/10.17533/udea.rfnsp.e337097

\section{Resumen}

Objetivo: Comprender los significados que los adolescentes le dan a la sexualidad. Metodología: Estudio cualitativo con enfoque etnográfico. La investigación se hizo con 17 adolescentes hombres, con un rango de edad entre los 12 y los 18 años, que tienen experiencia de vida en calle, en Medellín, Colombia. La información se recolectó por entrevistas y diario de campo. En el análisis se codificó y categorizó la información. Resultados: La sexualidad para los adolescentes con experiencia de vida en calle significa tener relaciones sexuales; estas se realizan por arrechera cuando, según ellos se les suben las hormonas, o por sobrevivencia, para suplir las necesidades de la vida cotidiana. Conclusiones: Para los adolescentes de la calle, el intercambio sexual con personas del mismo o de otro sexo significan sexualidad; las relaciones sexuales es algo cotidiano en ellos, están mediadas por el gusto, la necesidad fisiológica, el amor, el consumo de sustancias psicoactivas o por sobrevivencia.

-------Palabras clave: Sexualidad; adolescente; jóvenes sin hogar; antropología cultural; relaciones interpersonales.

* Este artículo es resultado de la investigación "Experiencias y significados de la sexualidad en los y las adolescentes con experiencia de vida en la calle Medellín" 


\begin{abstract}
Objective: To understand the meanings that adolescents give to sexuality. Methodology: A qualitative study with an ethnographic approach. The study was conducted with 17 adolescent males, with an age range between 12 and 18 years, who have experience of life on the street, in Medellin, Colombia. The information was collected through interviews and field diaries. In the analysis, information was codified and categorized. Results: Sexuality for adolescents with experience of life on the street means having sexual relations; these take
\end{abstract}

place due to arousal when, according to them, their hormones increase, or for survival, to meet the needs of everyday life. Conclusions: For street adolescents, sexual exchange with people of the same or opposite sex means sexuality; sexual relations are commonplace for them, they are mediated by taste, physiological need, love, consumption of psychoactive substances or for survival.

-Key words: Sexuality; adolescent; homeless youth; cultural anthropology; interpersonal relations.

\section{Resumo}

Objetivo: Compreender os significados que os adolescentes dão à sexualidade. Metodologia: Estudo qualitativo com foco etnográfico. A pesquisa foi feita com 17 adolescentes do sexo masculino na faixa etária de 12 a 18 anos, moradores de ruas em Medellin, Colômbia. A informação foi coletada através de entrevistas e pesquisa de campo. $\mathrm{Na}$ análise codificouse e caracterizou-se a informação obtida. Resultados: A sexualidade para os adolescentes moradores de rua significa ter relações sexuais que são realizadas por desejo quando segundo eles, se aceleram os hormônios, ou por sobrevivência para suprir as necessidades da vida cotidiana. Conclusões: Para os adolescentes da rua, o intercâmbio sexual com pessoas do mesmo sexo ou do sexo oposto significa sexualidade; as relações sexuais são algo normal para eles, estão medidas pelo prazer, pela necessidade fisiológica, pelo amor e pelo consumo de substâncias psicoativas ou por questões de sobrevivência. -Palavras-chave: Sexualidade; adolescente; moradores de rua; antropologia cultural; relações interpessoais.

\section{Introducción}

La sexualidad se encuentra presente en todo el curso de vida de las personas. El conocimiento sobre el significado de la sexualidad para los adolescentes con experiencia de vida en calle es importante, porque al igual que los otros adolescentes, pasan por esa etapa donde hay necesidades, desconocimientos, inquietudes y comportamientos alrededor de la sexualidad.

Para este trabajo, la adolescencia es definida como un grupo poblacional muy heterogéneo, que en una construcción sociocultural e histórica expresa un proceso desarrollado durante un momento de la vida, en la cual se configura y construye el paso de la infancia a la adultez $[1,2]$; representa una transición psicológica y desde este período se inician cambios biofisiológicos y psicológicos del desarrollo humano: los primeros, como estatura, peso, órganos sexuales, la primera eyaculación en los hombres, y los segundos, en los que se definen las preferencias sexuales y sociales, y se empiezan a diferenciar algunos sentimientos, como deseo, atracción sexual, enamoramiento, amor y apego.

Por otro lado, la sexualidad es entendida como una construcción social, histórica y cultural. Está relacionada con múltiples maneras en las que emociones, los deseos y las relaciones se expresan en los diferentes grupos culturales. La sexualidad existe en un contexto cultural, influenciada por distintos aspectos según el significado que se le dé [3]. Está encaminada al desarrollo de la vida y de las relaciones personales. Significa ser capaz de expresar y sentir placer [4]. López y Fuertes [5] afirman que la sexualidad es la función de relación con los demás y se caracteriza por la búsqueda de comunicación, afectividad y placer.

Para los adolescentes con experiencia de vida en calle, la sexualidad es importante y comienzan a experimentarla, en muchos casos, antes que los niños que no son de calle. Se constituye en un medio para conseguir dinero para subsistir y suplir necesidades cotidianas. Según estudios realizados, los niños y adolescentes inician la vida en la calle para buscar libertad $\mathrm{y}$, además, porque han sido agredidos física $\mathrm{y}$ sexualmente por parientes, vecinos y otras personas [6,7]. Existen trabajos sobre la sexualidad que incluyen las infecciones de transmisión sexual, el sida [8], la explotación o el abuso sexual, a los que se ven sometidos los adolescentes. Otras investigaciones, centradas en enfoques cuantitativos, muestran los problemas desde la mirada de los investigadores $[9,10]$. Y autores como Borges y Medeiros [8] y Catunda y Sousa [11] trabajan desde la mirada de los participantes. En ese sentido, en esta investigación se buscó conocer, desde la perspectiva de los adolescentes, sus experiencias relacionadas con la sexualidad y el significado que les dan a estas.

El acercamiento a la sexualidad de los adolescentes con experiencia de vida en la calle permitirá construir proyectos tanto de prevención de conductas que pongan en riesgo su integridad, como de educación sexual, de acuerdo con su realidad, y que generen impacto positivo en sus condiciones de vida, como un aporte 
a la construcción de espacios saludables y entornos protectores para ellos.

Dadas las circunstancias por las que atraviesa el país, como violencia, dificultades económicas de muchas personas, migraciones del campo a la ciudad e interurbanas, cada día se ven más adolescentes con experiencia de vida en la calle, y a pesar del interés de algunos autores por estudiar diversos aspectos relacionados con su sexualidad [12-15], desconocemos trabajos que se hayan realizado teniendo en cuenta la experiencia de los adolescentes. Esta investigación es pertinente por su vigencia y porque, como se mencionó anteriormente, hay pocos trabajos desde su propia mirada. Además, ellos mismos han expresado interés en conocer cuál es su situación frente a la sexualidad.

\section{Metodología}

La investigación es cualitativa, con enfoque etnográfico, y se usaron técnicas como la entrevista y el diario de campo, propias del trabajo etnográfico.

La investigación cualitativa se caracteriza por ser inductiva, al permitir que las categorías de análisis emerjan progresivamente durante la recolección de la información; se centra en la comprensión de significados o perspectivas de los actores, de la forma más amplia posible; busca identificar e interpretar los significados y las actividades cotidianas, para construir un sentido alrededor del fenómeno estudiado. Se caracteriza, según Patton [16], por ser holística, al plantear una interrelación entre el fenómeno de estudio y el contexto en el que se desarrolla.

El centro del enfoque etnográfico es la cultura, entendida por Geertz como "estructuras de significación socialmente establecidas" [17, p. 26]. Por lo tanto, la etnografía permite describir y analizar los procesos culturales dentro de un contexto. Según Geertz, es la "descripción densa" de la cultura y depende de la mirada de los actores - "mirada Emic" - [17], que se cumple cuando no es etnocéntrica y parte de la mirada del otro, sin desconocer los aportes teóricos y conceptuales del investigador [18].

El estudio se realizó con 39 adolescentes con experiencia de vida en la calle, 22 mujeres y 17 hombres, de la ciudad de Medellín durante los años 2016 y 2017. Dadas las diferencias que en el desarrollo de la investigación se encontraron respecto a la concepción de la sexualidad entre ambos géneros, fue publicado en un primer momento un artículo en el que se abordaron las significaciones de las mujeres [19].

En el proceso investigativo se hicieron reuniones con el propósito de generar confianza entre investigadores y participantes, de modo que al realizar las entrevistas, se generara la información requerida y se diera en un ambiente seguro para los participantes. Las entrevistas fueron grabadas con el consentimiento previo de los adolescentes y tuvieron una duración promedio de una hora cada una. Los investigadores llevaron diarios de campo.

El análisis incluyó la codificación de la información como proceso de caracterización y clasificación de los datos [20]; se construyeron categorías y subcategorías con base en los códigos obtenidos, y se realizaron mapas conceptuales para elaborar el informe final.

El principio de confidencialidad se tuvo en cuenta en la investigación, con el fin de generar un proceso de empatía con los participantes, optando de esta manera por mantener el respeto mutuo.

Los adolescentes se involucraron de forma voluntaria al proceso, luego de tener el conocimiento necesario y suficiente para decidir si participaban o no en la investigación. Así mismo, tenían la posibilidad de retirarse en cualquier momento, sin consecuencias.

Esta investigación fue aprobada por el Comité de Ética de la Facultad Nacional de Salud Pública en el Acta 141 del 29 de abril de 2016 y se ajusta a los requerimientos establecidos en la Resolución 008430 del Ministerio de Salud de Colombia, que regula la investigación con seres humanos en el país. Esta investigación se clasifica como de riesgo mínimo, ya que no implica ninguna modificación sobre la vida de los participantes [21].

El consentimiento informado se realizó de manera verbal, ya que los adolescentes no aceptan respaldar sus afirmaciones con firmas; se nombran a sí mismos con seudónimos y piensan que hacerlos firmar es muestra de desconfianza.

Se vinculó a los adolescentes en el análisis de la información para garantizar que esta fuera creíble, teniendo en cuenta lo dicho por Velasco et al., que para asegurar la credibilidad de la información es necesario establecer una buena relación con los entrevistados, generándoles confianza [20]. También, siguiendo a Castillo et al., la información se procesó al poco tiempo de haber sido recolectada, para asegurar la rigurosidad [22].

\section{Resultados}

Las zonas que frecuentan los adolescentes que hicieron parte de esta investigación están delimitadas en la comuna 10, con mayor afluencia en el parque de Bolívar, la plazuela de San Ignacio, la plaza Rojas Pinilla, la plaza de Zea, el parque de Boston, el parque de San Antonio, entre otros. Ocupan también barrios como El Sagrado Corazón de Jesús (Barrio Triste), Barrio Colón (Niquitao), San Benito, La Bayadera o San Diego, y lugares del centro de la ciudad frecuentados por 
habitantes de calle (jóvenes y adultos), como algunos puentes, el corredor del río Medellín, la plaza Minorista, el puente de la avenida Oriental con San Juan, la plaza de Flórez, el Museo de Antioquia, los alrededores de la iglesia de la Veracruz o Prado-Centro. En este último encuentran hoteles en donde pueden dormir y bañarse.

La sexualidad para los adolescentes con experiencia de vida en calle significa tener relaciones sexuales con personas de ambos sexos y se realizan por arrechera cuando, según ellos, se les suben las hormonas, o por sobrevivencia, cuando lo hacen para conseguir dinero y suplir las necesidades de la vida cotidiana.

Una práctica sexual, para mí, es tener relaciones con la mujer (Ernesto).

Los adolescentes buscan ser adultos; para ellos, un modo de lograrlo es salir de sus casas y formar una vida en la calle; la otra es conseguir una pareja para compartir, hacerla respetar y tener relaciones sexuales.

Yo de la edad como de trece años, ya tenía una mujer y la hacía respetar. A los 9 años, yo tuve la primera relación sexual (Saúl).

Algo que caracteriza a los adolescentes con experiencia de vida en calle es la desinhibición sexual, que responde al estímulo hormonal sin tener en cuenta las normas sociales; por lo tanto, no se cohíben a la hora de tener las relaciones sexuales.

Somos muy desinhibidos, no manejamos los prejuicios morales; entonces, por ejemplo, en la sexualidad, le damos rienda suelta a los instintos (Héctor).

Las relaciones sexuales implican interacciones entre hombre y mujer, y hombre y hombre, y en ellas pueden presentarse la confianza y la desconfianza, que les generan sentimientos como el miedo, el respeto, el rechazo y la felicidad.

\section{La arrechera}

Los adolescentes describen la arrechera como ganas de tener sexo, y se siente cuando, según ellos, se les suben las hormonas. La atracción se da por la excitación y como respuesta se espera que se dé la relación sexual; esta puede ser obligada, generalmente por violencia sexual y abuso hacia los menores, y no obligada, cuando hay mutuo acuerdo.

En el caso de algunos adolescentes, cuando inician la vida sexual, las mujeres les facilitan definir su preferencia sexual. En esta relación se da un proceso de exploración, tanto en aquellos que se consideran heterosexuales como en los que se asumen homosexuales, pero que quieren corroborar su condición $\mathrm{y}$, por esa razón, se acercan a las mujeres, tratando de ratificar su condición de homosexuales o, por el contrario, descubrir que pueden tener inclinación hacia las mujeres. El significado que le dan a la mujer varía según si el adolescente es homosexual o no; en el primer caso, la mujer tiene un significado de amiga o compañera, de persona muy linda, pero con la cual la relación no puede pasar del afecto; en el caso de los heterosexuales, la mujer puede representar atracción sexual.

Nacimos de una mujer; las quiero mucho, soy muy cariñoso con ellas y tengo muy buena amistad con ellas, pero como novia o mujer, no (Josué).

Las relaciones sexuales hombre-hombre y hombremujer generadas por la arrechera se dan por gusto, por necesidad fisiológica, por el amor o por el consumo de sustancias psicoactivas.

Las hormonas, es que no sé, que cualquier cosa lo pone arrecho a uno (John).

El gusto implica una atracción y responde a unas características que hacen llamativa a la persona. Cuando el gusto es por otros hombres, estos deben ser bonitos, limpios, no ser muy mayores y tener más de 17 años, porque los niños no les gustan; los adolescentes manifiestan tener desconfianza cuando los hombres son mayores o consideran que están enfermos.

Me gustan mucho los hombres de 17 o 18 años pa' arriba, porque verdaderamente son muy lindos, y yo adoro los hombres (Stiven).

En el caso de las mujeres, estas deben ser bonitas, bien vestidas, que no huelan maluco y que no sean de la calle, sino de barrio. Los adolescentes confían en las mujeres que pertenecen a los diferentes barrios de la ciudad, es decir, de las que están alejadas del centro o no permanecen en él. Este tipo de mujeres son las que prefieren para tener una relación estable, pero en algunos casos no lo logran, porque no se atreven a acercarse a ellas por vergüenza, generada por verse mal vestidos. También sienten desconfianza de las mujeres que consumen basuco (residuo que queda después de procesar la cocaína) y pegante (sacol), de las que son promiscuas o venden su cuerpo, de las mujeres mayores que ellos y de las que no son aseadas.

Porque hay peladas que son de barrio y peladas que no son de barrio y se prostituyen, y hay peladas de barrio que no se prostituyen; entonces, uno sabe con quién, con esas que se prostituyen, lo han hecho sin condón; entonces, hay que poner cuidado (Pedro).

Cuando es por necesidad fisiológica, no importa el gusto; prevalecen las ganas, y la relación sexual se lleva a cabo sin ninguna selección, ni precauciones.

Otra forma que los adolescentes consideran parte de la sexualidad por necesidad fisiológica es la masturbación, la cual significa descargarse por falta de sexo, una "bobada" que se puede superar o algo normal que se hace por estimulación. 
Veo porno y me dan ganas de masturbarme (Ernesto).

Está muy arrecho, "tan tan tan", se masturba y ya, se calma, porque queda todo encalambrado, ${ }^{\dagger}$ todo, todo maluco $[\ldots]$ (José).

La relación sexual por amor es ambivalente, puesto que aunque para ellos es fundamental, se constituye en un elemento que les genera incertidumbre. Los adolescentes afirman que para que haya una interacción estable debe haber amor. Este significa algo más que sexo e implica pensar más en la felicidad de la otra persona que en la propia; aunque el amor también puede ser la peor perdición y esto se da cuando se consideran traicionados y son abandonados. Para evitar esta situación, prefieren no establecer una relación por amor y buscan otras posibilidades.

Enamorarse de Dios, de la madre y de la plata que uno tenga en el bolsillo (Stiven)

El amor es una perdición (Raúl).

Los adolescentes refieren que se les aumenta el deseo sexual cuando consumen sustancias psicoactivas y tienen las relaciones bajo este efecto.

Yo no sé, yo me fumo un cripi ${ }^{\star}$ y quedo como yo no sé, con ganas de tiqui (Brian).

Las sustancias psicoactivas representan un elemento importante en la vida de los adolescentes; son utilizadas en casi todas las acciones propias de su cotidianidad y algunos llegan a afirmar que "lo primero es la droga" (Arturo). En el caso de la sexualidad, el uso de estas sustancias pueden ser consecuencia del rompimiento de una relación y ser utilizadas para olvidar. También se usan para tener seguridad en la forma de relacionarse, sentirse cómodos o tener una experiencia más placentera.

\section{Sobrevivencia}

Se denomina "sobrevivencia" cuando los adolescentes deben conseguir dinero para solucionar las carencias económicas y suplir las necesidades de la vida cotidiana, como pagar pieza, conseguir comida, comprar sustancias psicoactivas y recrearse. Por ello, los adolescentes plantean que la mejor opción para obtener dinero en el centro de la ciudad es tener relaciones sexuales; otra posibilidad que tienen es mediante la venta de sustancias psicoactivas, pero tratan de evitarlo, puesto que cuando trabajan en plazas de vicio pueden perder la libertad y ser señalados.

Encalambrado: quieto sin poder moverse (N. de los autores).

Cripa: Marihuana química más concentrada (N. de los autores).

Tiqui: sexo (N. de los autores)
Pues, acá en el Centro, prostituirse lo llaman un "trabajo", y ya lo que es trabajo en las plazas; pero no, yo no me hago calentar en una plaza, porque yo no quiero perder mi libertad (Josué).

En la interacción, negocian el dinero que les van a pagar según la solicitud y las exigencias del "cliente". Depende de esto decidir si acceden o no a dichas solicitudes.

Ya había tenido [sexo] con unas de allá abajo, con unos travestis homosexuales, y a veces por necesidad pongo a mamar a los cuchos; me da 20 o 30 mil pesos, a veces hasta 10 mil, y bueno, péguese pues. Usted sabe, uno como habitante de la calle le toca eso (Roberto).

La consecución de los "clientes" se hace mediante la negociación, que puede ser personal, telefónicamente o por medio de un tercero, como en el caso de la webcam, que se pacta un dinero de acuerdo con las funciones que debe cumplir el adolescente. Los lugares más comunes para conseguir personalmente los "clientes" son la calle y los parques.

Por dinero tenía que estar desnudándome en cámaras (Andrés).

La sexualidad por sobrevivencia se refiere, entonces, a la forma como los adolescentes consiguen dinero por medio de la explotación sexual comercial del cuerpo. Se realiza fundamentalmente con mujeres y hombres mayores, a quienes denominan "cuchos". Para los adolescentes, es reprochable que estos busquen satisfacerse con alguien menor que ellos.

Porque, ¿qué van a quitar las ganas con alguien menor que ellos? Con alguien sí... así como ellos, pero buscan alguien menor. ¿Pero sabe qué? Uno lo hace porque sí, le toca, porque eso es trabajo (John).

Las relaciones sexuales que sostienen con hombres, y especialmente con los denominados "cuchos", no los estimulan y, además, sienten aversión por el asco que les generan.

[...] cuando estoy con ellos ni siquiera sé... pues, no así como con una mujer, que tan, empieza a tocar y me pongo arrecho; no es lo mismo con hombres, antes me empieza a dar como ese asco (Esteban).

Las relaciones sexuales por sobrevivencia se diferencian de las que son por arrechera, en que no son deseadas. Cuando su preferencia es heterosexual y tienen relaciones sexuales con hombres, consideran que por esto no dejan de ser hombres y gustarles las mujeres.

Toca hacer eso, toca, y que uno, pues, ya lo penetra a uno, viendo que ni cuenta se dio... pues, ¿pero sabe qué? Eso no, uno no deja de ser hombre por eso, uno sigue siendo hombre, le siguen gustando las mujeres (Dairo). 
En algunos casos, los adolescentes reciben propuestas de mujeres adultas, quienes les ofrecen dinero, y como esto para ellos no es una situación recurrente, se sienten sorprendidos e intimidados cuando les sucede.

Esa señora muy adicta, pues, "le voy a dar diez mil", y yo: “¡iDiez mil!?”. Un ratico. Me agarró, yo quedé como todo tildado.**

yo me quedé así con los ojos abiertos y, bueno, pasó lo que pasó (Saúl).

\section{Sentimientos}

Las relaciones sexuales realizadas por arrechera o sobrevivencia generan, en los adolescentes, sentimientos como el miedo, el respeto, el rechazo y la felicidad; todos estos sentimientos los viven en el momento y no los comentan con los otros adolescentes que hacen parte de su círculo cercano.

El miedo puede ser generado por la otra persona. Se teme al desconocimiento y a que ese otro pueda ser portador de una infección de transmisión sexual. En ese desconocimiento evocan el pasado de la otra persona, es decir, que se relacionan también con la historia.

Uno se acuesta con el pasado de la otra persona (Raúl).

El miedo disminuye cuando se logra conocer al otro, porque baja la noción del riesgo, aumenta la confianza, se relajan y no se protegen. En este momento, no se tiene en cuenta el pasado de la otra persona y la posibilidad de que esta puede transmitir una infección es dejada de lado.

Yo veo una mujer y yo no sé, como que me la quiero, tin, comer, y yo veo cualesquiera y de una, y me dan es como ganas. Y hay días que no hay forrito [condón]; entonces, toca así, y muchas enfermedades por ahí, y usted sabe, uno en la calle como un guerrero, toca esas vueltas (Cristian).

Cuando los adolescentes que van a tener una relación sexual con cualquier persona hacen un análisis que comprende el conocimiento que tenga de ellas, la experiencia previa y la confianza que les genera, si después de hacer este análisis sienten miedo porque consideran que pueden infectarse, se protegen mediante el uso del preservativo o condón. En caso de no sentir miedo, entonces no se protegen y esto lo hacen con el fin de llegar a sentir mejor la sensación en la relación sexual. Un elemento importante es que la estabilidad

** Quedar tíldado: asombrado, sin palabras (N. de los autores).

$\dagger$ Sacolerita: consumidora de sacol (N. de los autores)

* Ruedas: pastillas que tienen efectos psicoactivos (N. de los autores) en la relación, la cercanía y el gusto que tengan por la pareja disminuyen la sensación de riesgo.

Nos tenemos que cuidar con el sexo, con un condón, o si se sabe que la pareja es limpia (Andrés).

Uno compra su condón o depende la pelada que sea (Esteban).

Los adolescentes no hacen análisis de las personas con quienes van a tener la relación, fundamentalmente por dos razones: 1) cuando no tienen la posibilidad de pensar por la necesidad fisiológica o porque están "amurados", es decir, cuando están dominados por la droga; y 2) cuando no tienen dinero para comprar el preservativo o condón.

A veces todo "amurado", le toca es comerse cualquier sacolerita ${ }^{\dagger \dagger}$ por ahí (Dairo).

El respeto para los adolescentes es como la ley del silencio en la calle, que consiste en no hablar de las personas con las que se han tenido las relaciones sexuales ni de las circunstancias en las cuales estas se dan, por su propia reputación y la de las otras personas. La ley del silencio también aplica para las circunstancias que se les presentan en su vida diaria.

Una ley: ciegos, sordos y mudos, eso es como una ley de la calle (Cristian).

El rechazo gira en torno a no querer tener relaciones sexuales con personas que no les generan atracción sexual, pero como reciben dinero por ello, las tienen que realizar para sobrevivir en la calle y usan sustancias psicoactivas para no ser conscientes de sus actos, olvidar y desconectarse.

Por eso, yo más bien, cuando voy a estar con un hombre... las ruedas, $\$$ las ruedas para ponerme loco y no saber lo que voy a hacer (Arturo).

La felicidad es efímera. En todas las relaciones sexuales, los adolescentes sienten felicidad, excepto cuando deben realizarlas por obligación (sobrevivencia) y la definen como un rato agradable que pasa y se olvida.

Como un rato feliz y ya después del rato feliz, ahí quedó todo (Josué).

\section{Discusión}

Los hallazgos de este trabajo en lo referente a la sexualidad, entendida como la relación sexual, coinciden en lo encontrado en los estudios de Borges y Medeiros [8], Catunda y Sousa [11] y Medeiros, Carvalho, Bouttelet y Gomes [23], quienes realizan investigación con adolescentes en situación de calle. 
Además, Márquez y Marín [24] encontraron que la sexualidad es entendida como genitalidad.

A diferencia de lo que se piensa tradicionalmente, el hecho de estar en la calle no cohíbe a los adolescentes de tener relaciones sexuales, según lo hallado en nuestro estudio y que coincide con Medeiros et al. [23]. Las relaciones sexuales se realizan respondiendo a estímulos que reciben diferentes nombres, como "arrechera" [25], "respuesta hormonal" [26] y "búsqueda de placer" [10]. Cuando estas relaciones no son posibles, los adolescentes acuden a la masturbación para satisfacer esa necesidad fisiológica [27].

Gaete plantea, en su estudio, que los adolescentes se preocupan por sus cuerpos y los cambios que se producen en la etapa de la pubertad [27]. En este trabajo se encontró que los hombres no manifiestan preocupación por los cambios del cuerpo.

Al igual que lo hallado en este trabajo, en relación con la venta del cuerpo por parte de los adolescentes que habitan en la calle para la compra de droga, comida y pagar la pieza, entre otras, Márquez y Marín [24] encontraron esta misma situación en estudios realizados en la comuna 10 de Medellín y Salvidia, en Santiago de Chile [28].

Los adolescentes en situación de calle consumen sustancias psicoactivas para desinhibirse, no rechazar a los clientes, evadir la realidad [24] y así no tener perjuicio emocional cuando realizan el intercambio de dinero por sexo. Esto concuerda con lo encontrado en el estudio "Realidades de las niñas y adolescentes en explotación sexual del sector Prado Centro de la comuna 10 de Medellín" [29]. Igualmente, los autores refieren que los adolescentes no consumen droga para no tener riesgo cuando "hacen los ratos" (es decir, venden su cuerpo), pero en este estudio se halló que los adolescentes, aunque identifican el riesgo, no dejan de consumir cuando están con los clientes.

Los adolescentes conocen los riesgos, pero responden a una serie de estímulos, como las recompensas, que se incrementan en presencia de otros adolescentes, según un estudio sobre el neurodesarrollo realizado por Konrad, Firk y Uhlhaas [30].

Borges y Medeiros [8] y Medeiros et al. [23] plantean la importancia de la confianza para tener una relación estable. Para Medeiros et al. [23], al igual que en este estudio, dicha relación se da preferiblemente con adolescentes que sean de barrios y no habiten en la calle.

Delemeau plantea que el miedo es suscitado por la gente desconocida, mal conocida, que no vive del mismo modo y tiene códigos distintos, cuya significación no se entiende. A los adolescentes con experiencia de vida en calle, el desconocido les produce miedo, porque no conocen su historia [31]

Según Padrós, Soria y Navarro [32], la afectividad guía el pensamiento y el comportamiento, los organiza, funciona como un motor y a veces como freno. Esto coincide con lo expresado por los adolescentes, para quienes tanto las relaciones sexuales realizadas por arrechera o sobrevivencia son el producto del estímulo generado por sentimientos que impulsan su respuesta sexual.

El amor genera proximidad y apego, se percibe el mundo de otra manera, se construye una "realidad" diferente. El miedo provoca un distanciamiento $\mathrm{y}$, eventualmente, la huida o la defensa; la rabia establece unos límites. Los afectos son indispensables para comprender el mundo y la realidad cotidiana que nos circunda [33].

\section{Conclusiones}

Para los adolescentes con experiencia de vida en calle, la sexualidad significa tener relaciones sexuales con personas de ambos sexos y se realizan por arrechera (ganas), en donde median gusto, necesidad fisiológica, amor o consumo de sustancias psicoactivas; o por sobrevivencia (explotación sexual comercial), lo que permite obtener dinero más fácil con respecto a otras actividades.

Cuando el intercambio sexual se hace por dinero, los adolescentes incrementan el consumo de sustancias psicoactivas, para no darse cuenta al momento de tener el encuentro sexual.

La protección que realizan para evitar el contagio de enfermedades de transmisión sexual depende del miedo que resulte del análisis que se hace de la pareja, del dinero que tengan para comprar el condón, y de si están en medio de una situación de tensión sexual, ya sea por el consumo de sustancias psicoactivas o por estar con una persona que les genera atracción y no cuentan con preservativos en el momento.

El panorama que emerge para el abordaje de la sexualidad debe hacerse a la par con la prevención, la mitigación y la reducción de riesgos frente al consumo de sustancias psicoactivas, $y$ debe permitir una construcción en la que los adolescentes sean partícipes de las políticas que se establecen en la ciudad, para dar respuesta a las necesidades que estos tienen. Asimismo, acompañarles en sus contextos, dignificando la condición en la que se encuentran, sin que se violente su elección de vida inmediata, e implementar espacios de escucha y acciones que trasciendan la entrega de un preservativo.

El conocimiento que se tiene sobre sexualidad permite la prevención de situaciones que afectan la salud física y mental, y la forma como los adolescentes se relacionan con el entorno; y, específicamente, cómo se protegen de las enfermedades de transmisión sexual y de la violencia sexual. 


\section{Agradecimientos}

A los adolescentes en situación de calle, por su participación activa y constante en la investigación.

A los estudiantes y docentes que hacen parte de la "Línea de Investigación Cultura y Salud", de la Facultad Nacional de Salud Pública, por su apoyo en todo el proceso.

A la Universidad de Antioquia, y a las Facultades Nacional de Salud Pública y Enfermería.

\section{Conflicto de intereses}

Los autores declaran no tener conflictos de intereses relacionados con la investigación y el artículo producto de ella.

\section{Financiación}

Este artículo hace parte de la investigación "Experiencias y significados de la sexualidad en los y las adolescentes con experiencia de vida en la calle Medellín", INV 526 - 16, que es financiado por el Fondo de Apoyo Docente de la Facultad Nacional de Salud Pública, Universidad de Antioquia.

\section{Declaración de responsabilidades}

Se declara que los puntos de vista expresados en todo el artículo son responsabilidad de los autores y no de la institución a la que pertenecen los autores, ni de la institución en la cual se encuentra registrado el proyecto del cual sale este artículo, ni tampoco de la institución que financió dicho proyecto.

\section{Declaración de autoría}

Los autores declaran cumplir con los criterios de International Committe of Medical Journal Editors (ICMJE): participaron de manera equitativa en las etapas de elaboración del proyecto, recolección y análisis de la información, y escritura del artículo.

\section{Referencias}

1. Checa S. Aproximaciones a la problemática de la sexualidad adolescente. En: Checa S, compiladora. Género, sexualidad y derechos reproductivos en la adolescencia. Buenos Aires: Paidós; 2003. pp. 19-36.

2. Villa A. Cuerpo, sexualidad y socialización. Intervenciones e investigaciones en salud. México: Noveduc; 2007.

3. Dides C, Benavente C, Morán J. Estado de la vinculación de la salud sexual y reproductiva, VIH y sida e ITS en Chile. Chile:
Organización Panamericana de la Salud y Programa de Género y Equidad de FLACSO-Chile, UNFPA; 2009.

4. Vera L. Historia de la sexualidad. Rev. Bioméd. 1998;9(2):116-21.

5. López F, Fuertes A. Para comprender la sexualidad. Navarra: Verbo Divino; 1991.

6. Giraldo Á, Forero C, López A, et al. Cómo vivimos los niños y las niñas en la calle. Medellín: Cátedra Litografía; 2016.

7. Brasesco, M. Niños de la calle: Buenos Aires SXXI. Cienc. Psicol [internet]. 2011 [citado 2018 jun. 18]; 5(1);7-18.Disponible en: http://www.scielo.edu.uy/scielo.php?script=sci_arttext\&pid $=$ S1688-42212011000100002

8. Borges I, Medeiros M. Representações sociais de DST/AIDS para adolescentes de uma instituição abrigo com experiência pregressa de vida nas ruas da cidade de Goiânia. DST - J bras Doenças Sex Transm. 2004; 16(4): 43-49.

9. Abreu R, Reyes O, García G, et al. Adolescencia e inicio precoz de las relaciones sexuales. Algunos factores desencadenantes. Gaceta Médica Espirituana[internet]. 2008 [citado 2018 jun. 18]; 10(2):1-8. Disponible en: http://revgmespirituana.sld.cu/index. $\mathrm{php} / \mathrm{gme} /$ article/view/1239

10. Cid M, Pérez R, Katia S. Orientación sexual en los adolescentes y su asociación con variables demográficas, socioconductuales y sexuales. Perinatol Reprod Hum. 2011;25(2):74-80.

11. Catunda L, Sousa S de. A sexualidade de adolescentes masculinos com experiência de rua e em situação de abrigo [tesis doctoral]. [Fortaleza]: Universidade Federal do Ceará, Faculdade de Educação, Programa de Pós-Graduação em Educação Brasileira; 2009.

12. Villaseñor P, García J. Actitudes hacia la sexualidad en adolescentes en situación de calle. Archivos Hispanoamericanos de Sexología Universidad Nacional de México. 2003;9(1):105-23.

13. Agudelo L, Garzón D. ¿Qué significado le atribuyen a la vivencia de su sexualidad y a la construcción de su identidad las y los jóvenes entre 13 y 20 años que están en situación de calle en el centro de la ciudad de Medellín durante el año 2011? [trabajo de grado]. [Medellín]: Universidad de Antioquia, Facultad de Ciencias Sociales y Humanas; 2012.

14. Muñoz I, Noreña C, Londoño B, et al. Morbilidad atendida y conductas de riesgo de la niñez y adolescencia en situación de calle de Medellín, 2008. Revista de Salud Pública. 2008;13(2):207-18. DOI: https://doi.org/10.1590/S0124-00642011000200003

15. Moreno A. Salud sexual y reproductiva en adolescentes callejeras. Temas sociales. 2015;(25):45-57.

16. Patton M. Qualitative research and evaluation methods. 3.a ed. Beverly Hills: Sage Publications; 1990.

17. Geertz C. La interpretación de las culturas. Barcelona: Gedisa S. A.; 2003.

18. Reguillo R. De la pasión metodológica o de la (paradójica) posibilidad de la investigación. En Mejía R, Sandoval S, coordinadores. Tras las vetas de la investigación cualitativa. Perspectivas y acercamientos desde la práctica. Guadalajara: ITESO; 2003. pp. 17-38.

19. Giraldo Á, Forero C, Posada LF. Sexualidad es tener sexo: las adolescentes en situación de calle. Index Enferm [internet]. 2017 [citado 2018 ago. 21]; 26(1-2):15-19. Disponible en: http://scielo.isciii.es/scielo.php?script=sci_arttext\&pid=S1132$12962017000100004 \& \operatorname{lng}=\mathrm{es}$

20. Velasco H, Díaz A. La lógica de la investigación etnográfica. Un modelo de trabajo para etnógrafos de escuela. 4.a ed. Madrid: Trotta; 2014. 
21. Colombia, Ministerio de Salud. Resolución 008430, por la cual se establecen las normas científicas, técnicas y administrativas para la investigación en salud (1993 oct. 4).

22. Castillo E, Vásquez M. El rigor metodológico en la investigación cualitativa. Colombia Médica. 2003;34(3):164-7.

23. Medeiros M, Carvalho M, Bouttelet D, et al. A sexualidade para o adolescente em situação de rua em Goiânia. Rev Latino-am Enfermagem. 2001;9(2):35-41. DOI: https://doi.org/10.1590/ S0104-11692001000200005

24. Márquez C, Marín M. Significado de la explotación sexual comercial en el contexto de la vida en calle y de la calle, para cuatro (4) adolescentes entre 14 y 17 años, en dos sectores de la comuna 10 de Medellín, 2014 [tesis de pregrado]. [Medellín]; Corporación Universitaria Minuto de Dios UNIMINUTO, Programa de Psicología Facultad de Ciencias Humanas y Sociales; 2015.

25. Giraldo Á, Forero C, López A. Aprender la sexualidad desde la "arrechera": los y las adolescentes en situación de calle. En Costa A, Tuzzo S, Brandão C, editores. Investigação Qualitativa em Ciências Sociais. Brasil; 2017. pp. 59-67.

26. Master W, Johnson V. Respuesta sexual humana. Buenos Aires: Intermédica; 1967.

27. Gaete V. Desarrollo psicosocial del adolescente. Rev Chil Pediatr. 2015;86(6):436-43. DOI: http://dx.doi.org/10.1016/j. rchipe. 2015.07 .005
28. Saldivia L. Adolescentes en situación de calle: construcción de identidad en situación de extrema vulnerabilidad. Un acercamiento cualitativo [tesis de maestría]. [Santiago de Chile]: Universidad de Chile, Facultad de Ciencias Sociales, Escuela de Post Grado; 2005

29. Convenio de Asociación Municipio de Medellín, Secretaría de las Mujeres y Corporación Amiga Joven. Realidades de las niñas y adolescentes en explotación sexual del sector Prado Centro de la comuna 10 de Medellín. Medellín; 2012.

30. Konrad K, Firk C, Uhlhaas P. Brain development during adolescence, neuroscientific insights into this developmental period. Dtsch Arztebl Int. 2013;110(25):425-31. DOI: https://doi. org/10.3238/arztebl.2013.0425

31. Delemeau J. Miedos de ayer y de hoy. En Delemeau J, Uribe M, Giraldo J, et al. El miedo. Reflexiones sobre su dimensión social y cultural. Medellín: Corporación Región; 2002. pp. 9-24.

32. Padrós F, Soria C, Navarro G. Afecto positivo y negativo: ¿una dimensión bipolar o dos dimensiones unipolares independientes? Interdisciplinaria. 2012;29(1):151-64.

33. Ciompi L. Sentimientos, afectos y lógica afectiva. Su lugar en nuestra comprensión del otro y del mundo. Rev. Asoc. Esp. Neuropsiq. 2007;27(2):425-43. 